\title{
Optical lattice clock on bosonic Strontium atoms
}

\author{
N. Poli , M. G. Tarallo ${ }^{1,2}$, M. Schioppo ${ }^{1}$, D. Sutyrin ${ }^{2}$, N. Beverini ${ }^{2}$, C. W. Oates ${ }^{3}$ and G. M. Tino \\ 1. LENS and Dipartimento di Fisica, INFN-Sezione di Firenze, Università di Firenze, \\ Via N. Carrara, 1 -50019 Sesto Fiorentino-Italia, E-mail:poli@lens.unifi.it \\ 2. Dipartimento di Fisica, Università di Pisa, Largo B. Pontecorvo, 3 - 56100 Pisa, Italia \\ 3. National Institute of Standards and Technology, Boulder, Colorado 80305 USA
}

We report on recent realization of an optical lattice clock operating on the ${ }^{1} \mathrm{~S}_{0}-{ }^{3} \mathrm{P}_{0}$ transition in ${ }^{88} \mathrm{Sr}$, at LENS laboratories. The clock transition is excited with the technique of magnetic field-induced spectroscopy ${ }^{1}$. New experimental techniques has also been developed in order to simplifies the clock spectroscopy in $\mathrm{Sr}$ atoms ${ }^{2}$. The first new method helps in the first search of the clock transition without the use of extensive frequency metrology hardware and in particular optical frequency combs. This technique exploits a near coincidence in the atomic wavelengths of the ${ }^{1} \mathrm{~S}_{0}{ }^{3} \mathrm{P}_{0}$ clock and ${ }^{1} \mathrm{~S}_{0}{ }^{3} \mathrm{P}_{1}$ second stage cooling transitions in $\mathrm{Sr}$, which are only $5 \mathrm{THz}$ far apart. This coincidence enables the use of an optical (transfer) cavity to reference the frequency of the clock transition relative to that of the much stronger cooling transition. Secondly we reduce the complexity of the experimental setup by using only semiconductor laser sources in the apparatus ${ }^{3}$. With this setup, about $10^{4}{ }^{88} \mathrm{Sr}$ atoms are trapped in a 1D lattice formed by $200 \mathrm{~mW}$ of radiation tuned near the magic wavelength at $813 \mathrm{~nm}$.

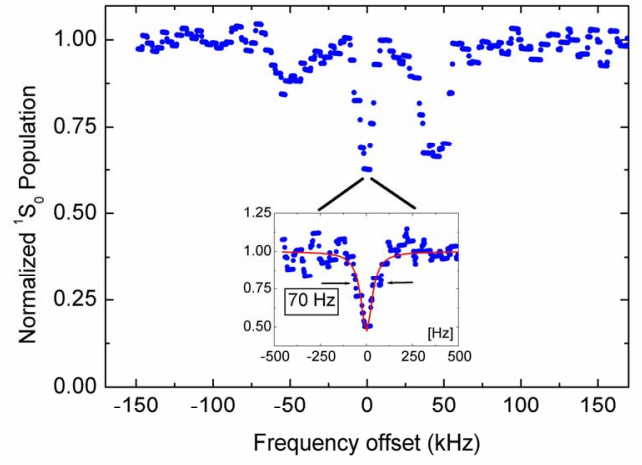

Fig. 1 Spectroscopy of clock transition for ${ }^{88} \mathrm{Sr}$ atoms trapped in $1 \mathrm{D}$ lattice at magic wavelength. In the inset is shown the central peak at highest resolution showing a $70 \mathrm{~Hz}$ linewidth with $\mathrm{S} / \mathrm{N}$ of about 7.

We will present a first uncertainty budget for our optical lattice clock based on ${ }^{88} \mathrm{Sr}$ with particular attention to density dependent collisions, which led to an unexpectedly high signal contrast for long interaction times. In view of an absolute frequency measurement of clock transition we'll also present recent experimental results on optimization of an home made Ti:Sa optical frequency comb. Additionally, we anticipate that the simplification of the experimental setup presented will help address a wider range of applications including those requiring transportable devices. Along these lines we will report progress on the realization of the first transportable Sr optical lattice clock ${ }^{4}$.

\section{References}

[1] A.V. Taichenachev, et al., "Magnetic Field-Induced Spectroscopy of Forbidden Optical Transitions with Application to Lattice-Based Optical Atomic Clocks", Phys. Rev. Lett. 96, 083001 (2006).

[2] N.Poli et al., "A simplified optical lattice clock", arXiv:0812.4259v1, submitted to Appl. Phys. B

[3] N. Poli, R. E. Drullinger, M. G. Tarallo, and G. M. Tino, "Strontium optical lattice clock with all semiconductor sources", Frequency Control Symposium, 2007 Joint with the 21st European Frequency and Time Forum. IEEE International, 655, (2007)

[4] N. Poli et al., "Prospect for a compact strontium optical clock", Proc. SPIE 6673, 66730F (2007) 